\title{
Onion: Anti Cancer Sulfur Compounds with High Cancer Chemo Prevention Potentials
}

\author{
Dibyajyoti Saha
}

Department of Pharmacy, BGC Trust University Chittagong, Bangladesh

\begin{tabular}{|c|c|}
\hline Abstract & Article Information \\
\hline \multirow{3}{*}{$\begin{array}{l}\text { Onions are chock full of anti cancer sulfur compounds which are highly beneficial } \\
\text { for cancer chemoprevention. Advanced studies have shown that onion may be } \\
\text { inhibit liver, colon, prostate, lung cancer etc. with high antioxidant properties which } \\
\text { also reduce not only oxidative stress, free radicals but also xenobiotics. Green } \\
\text { onion is highly beneficial for women to prevent ovarian cancer where white onions } \\
\text { are not effective for prevention of cancer. This communication suggested the briefly } \\
\text { possible mechanism of action of various onions and also illustrate the techniques to } \\
\text { cut an onion to maximize anti-cancer compounds and minimize eye irritation. }\end{array}$} & $\begin{array}{l}\text { Article History: } \\
\text { Received : } 01-08-2013\end{array}$ \\
\hline & \\
\hline & $\begin{array}{l}{ }^{*} \text { Corresponding Author: } \\
\text { E-mail: } \\
\text { saha.dibyajyoti@gmail.com }\end{array}$ \\
\hline
\end{tabular}

\section{LETTER}

Cancer chemoprevention is defined as the use of pharmacological, natural or dietary agents to inhibit the development of invasive cancer by blocking DNA damage caused by carcinogens or by arresting the progression of premalignant cells after damage has already occurred (Saha et al., 2011a). The genetic analysis of cancer is advancing rapidly as a result of recent advancement of techniques which allow study of the sequence of the DNA and the cloning, manipulation of genes (Saha et al., 2011b). In the treatment of cancer, one of the basic assumptions is that all malignant cells should be destroyed, removed or neutralized to achieve cure (Saha et al., 2011c). Xenobiotics are chemical compounds foreign to the body; such as drugs, food additives, environmental pollutants; more than 200,000 have been identified and they are metabolized into two phases. Oxidative stress is a large increase in the cellular reduction potential, or a large decrease in the reducing capacity of the cellular redox couples. Free radicals cause a chain reactions leading to consecutive oxidation. These radicals attack molecules like fat, protein, DNA, sugar etc. Antioxidants are beneficial components that neutralize free radicals before they can attack cell proteins, lipids and carbohydrates. The mechanism involves significant inhibition or delay in the oxidative process. Biochemist \& epidemiologists have stated the antioxidants neutralize free radicals by binding their unpaired electrons and rendering them harmless (Saha et al., 2011d).
Although decreasing cancer incidence and mortality is the ultimate goal of chemoprevention, much effort is being devoted towards identifying specific steps in the carcinogenesis process that are amenable to intervention and that can serve as surrogate endpoint biomarkers for possible modulation by chemopreventive agents. There appear to be at least two basic mechanisms by which chemical agents with relatively low toxicity may inhibit carcinogenesis. Chemopreventive agents can also identified by systematic evaluation of agent that acts at specific molecular targets with respect to cancer by using laboratory assay (Saha et al., 2011e). Botanicals have been used for the treatment of various human diseases throughout history. In addition, botanicals play a role in disease prevention. For example, epidemiologic studies have suggested that a reduced risk of cancer is associated with high consumption of vegetables and fruits. The allium genus includes approximately 500 species. Commonly used allium vegetables include garlic, onion, leeks, chives, scallions which are used all over the world in different delicacies. Some allium vegetables have been employed for millennia in the traditional medical practice to treat cardiovascular diseases. They have been shown to have applications as antimicrobial, antithrombotic, antitumor, hypolipidaemic, antiarthritic and hypoglycemic agents. Garlic (Allium sativum) and onion (Allium cepa) are among the oldest of all cultivated plants and now used as a food and for medical purpose. 


\section{Dibyajyoti Saha}

\section{ONION AS CANCER CHEMOPREVENTIVE}

Varieties of onions are chock full of anti-cancer chemicals. However, certain varieties are particularly high in these compounds as well as more effective in inhibiting liver and colon cancer cell growth. According to a new study by Cornell University food scientists; Shallots, Western Yellow, pungent yellow and Northern Red onions are higher in anti-cancer chemicals than other varieties tested. Furthermore, Shallots and Western Yellow and pungent yellow onion varieties are particularly effective against liver cancer cells, while pungent yellow and Western Yellow varieties have the greatest effect on colon cancer cells. Ten onion varieties and shallots clearly shows that onions and shallots have potent antioxidant and antiproliferation activities and that the more total phenolic and flavonoid content an onion has, the stronger its antioxidant activity and protective effect. Phenolics and flavonoids are types of phytochemicals antioxidant chemicals that protect plants against bacteria, viruses and fungi. Phenolics and flavonoids, like other antioxidants, help prevent cancer by mopping up cell-damaging free radicals and inhibiting the production of reactive substances that could damage normal cells. Scientists found that shallots had six times the phenolic content of the onion variety with the lowest content (Vidalia). Western Yellow onions had eleven times more flavonoids than Western Whites, the onions with fewest flavonoids. Using colon cancer cells treated with extracts from the ten onion varieties and shallots that were tested, it was found that pungent yellow and Western Yellow onion extracts provided the strongest anti-proliferation protection against colon cancer cells. The Western Yellow, shallot and pungent yellow extracts provided the strongest antiproliferation protection against liver cancer cells. Onions are one of the richest sources of flavonoids in the human diet, and flavonoids consumption has been associated with a reduced risk of cancer, heart disease and diabetes. Flavonoids are not only anticancer but also are known to be anti-bacterial, antiviral, anti-allergenic and anti-inflammatory. Onions and leeks offer the best protection against prostate cancer, while eating meat is the biggest risk factor for the disease, according to new research. Researchers now believe that about one-third of cancer cases could be prevented by diet, the National Cancer Institute and American Cancer Society recommend that Americans consume at least five servings of fruits and vegetables a day. A number of other studies have found that phytochemicals \& antioxidants are the chemicals in fruits and vegetables that could be effective against cardiovascular disease and certain cancers, such as lung cancer, prostate cancer \& melanoma (Galeone et al., 2006). Onions, garlic, \& their family members also contain flavonoids \& phenols. White onions are
Sci. Technol. Arts Res. J., July-Sep 2013, 2(3): 01-02

not as rich in these antioxidant compounds as yellow and red, and shallots are especially high in polyphenol levels. Red onions are particularly rich in anthocyanins and quercetin. Green onions, like all onion varieties, contain powerful substances that scientists believe may help fight cancer and other diseases. Green onions have a mild taste that is not overwhelming. Because they will not dominate the flavor of a dish, they are commonly used in salads, sandwiches, soups and stir-fry dishes. Green onions can be eaten raw or cooked. The flavonoids in green onions also benefit health by preventing certain cancers from developing. Quercitin, a flavonoid in green onions, will slow the formation of cancer cells and precancerous lesions in the colon. Women can particularly benefit from eating green onions. The flavonoid, kaempferol, in onions is known to lower the risk of ovarian cancer. Onions and the other vegetables of the allium family can be added to any and every vegetable dish for great flavor and anti-cancer benefits. Remember that they must be eaten raw and chewed well or chopped finely before cooking to initiate the chemical reaction that forms the protective sulfur compounds.

\section{Techniques to Cut an Onion to Maximize Anti-} Cancer Compounds and Minimize Eye Irritation

The onion must be cold before cut it, even putting the onion in the freezer for five minutes is sufficient. It can use a fan to blow the gaseous compounds away. Cut the end of the root off with the root facing away and preserving as much of the onion adjacent to the root as possible. The root is the part of the onion with the highest concentration of these anti-cancer compounds. Then cut or chop the onion finely, slice thinly, or put it in a food processor before adding to soup, salad, or vegetable dish to maximize the production of sulfur compounds.

\section{REFERENCES}

Galeone, C., Pelucchi, C., Levi, F. (2006). Onion and garlic use and human cancer. American Journal of Clinical Nutrition 84:1027-1032.

Saha D., Nath, A.K., Uddin, M.Z., Dutta, M. (2011e). Cancer Chemoprevention: Boon for Cancer Biology. Journal of Dhaka International University. 3(1):140-45.

Saha, D., Maity, T., Jana, M., Mandal, S. (2011c). Cancer Treatment Strategy-An Overview. Asian Journal of Pharmaceutical Technology 1 (2): 28-33.

Saha, D., Mridha, D., Beura, S. (2011a). Nano Selenium: Potential Boon and Novel Approaches for Cancer Chemoprevention. Research Journal of Pharmacology and Pharmacodynamics. 3(1): 15-16.

Saha, D., Mridha, D., Mondal, S., Jana, M., Kayal, S. (2011b). Organoselenium as a Cancer Chemopreventive agent against Carcinogenesis. Research Journal of Pharmacy and Technology 4(3): 367-368.

Saha, D., Tamrakar, A. (2011d). Xenobiotics, Oxidative Stress, Free radicals Vs. Antioxidants: Dance of Death to Heaven's Life. Asian Journal of Research in Pharmaceutical Sciences 1 (2): 36-38. 